\title{
Ant Colony Optimization and Road Transportation Route of Dangerous objects
}

\author{
HenghaiZhang1,3,Jianyou Zhao2, DonglingXiao4,Meng Lei5 \\ 1School of Automotive Engineering, Shan Dong Jiaotong University, Jinan, 250023, China; \\ 2School of Automobile, Chang'an University, Xian 710064, Shaanxi, China \\ 3State Key Laboratory of Automotive Simulation and Control, Jilin University, Changchun, 130022,
} China 4Automotive Department, Henan Vocational and Technical College of Communications,
Zhengzhou, 450005, China

5Auto Maintenance Technology Department, Hangzhou Technician Institute, Hangzhou, 311500,China

Keywords: Routeoptimization, Ant colony Optimization(ACO),Dangerous, objects, MATLAB

\begin{abstract}
According to the problem of dangerous objects transportation route optimization, using the colony algorithm theory as a guide, making analyses on the degree of risk road calculation model,developing ACO, then writing in MATLAB transport of dangerous objects route optimization program,and validating in road transport of general cargo vehicle routing problem and road transport of dangerous objects vehicle routing problem. The results indicate that under the same parameters, due to the risk of the road value, the road transport of dangerous objects vehicle route simulation line with general cargo transportation vehicle paths are different.
\end{abstract}

\section{Introduction}

Dangerous goods transportation affects human life and environment if accidents occur in high-density living environments.Effective dangerous objects route minimizes the societal risks related to their movements, andbecome the cost efficient solution for all actors involved in their logistic chain.

Vehicle routing by ACO is an important logistics problem, which has been studied for several decades. John E. Bella and Patrick R. McMullen apply the meta-heuristic method of ACO to an established set of vehicle routing problems[1]. ArdavanAsef-Vaziri et al. develop a global optimization model, simplification schemes, and a heuristic procedure for the design of a shortcut-enhanced unidirectional loop aisle-network with pick-up and drop-off stations[2]. S.R. Balseiro et al. present an Ant Colony System algorithm hybridized with insertion heuristics for the Time- Dependent Vehicle Routing Problem with Time Windows[3]. Christopher L. Fleming et al. takes an initial look into the influence of triangle inequality violations on vehicle routing problems[4].

Route planning of dangerous goods has been an important problem in the field of distribution and logistics since at least the early 1980s. Abkowitz, M and Cheng, P Ddescribe a methodology which has been developed that incorporates risk and cost into a framework for optimizing the routing of truck movements of dangerous goods[5]. P. Leonelli et al. introduce a methodology based on risk analysis for the selection of the best route for the transport of a hazardous substance[6], whereas this procedure involves the calculation of risk indexes suitable for linear risk sources. WL $\mathrm{Ng}$ et al. present a case study on a tanker assignment and routing problem for petrol products in Hong Kong[7]. Li Zhi et al. establish the mathematical model about the vehicles routing problem (VRP) of transporting dangerous goods in Zhengzhou Coal Material Supply and Marketing Company, and use artificial fish swarm algorithm to explore the optimal solution of the VRP[8]. Li, Rongrong et al. develop a multiobjective genetic algorithm(MOGA) for the determination of optimal routes for dangerous goods transportation under conflicting objectives [9].

Weimprove ACO, applydangerous objects transportation route, and compare with general goods 
transportation route.

\section{Optimization Design}

The concept of risk is the relation between frequency and the number of people suffering from a specified level of harm in a given population from the realization of specified hazards [10].To collect data easily, a large number oftraffic accidentsdetermine the quantity of dangerous objects transport accident probability, which can be more appropriate for risk assessment on transportation.Degree of road risk model is expressed as follows:

$$
R_{\mathrm{ijh}}=\sum_{i=1}^{n}\left[F_{\mathrm{ij}} \times V_{i j h} \times\left(r_{i j h}+T_{i j}\right)\right](1)
$$

Where $R_{\mathrm{ijh}}$ is the degree of road risk, iis the current location on the path, jis the next possible location on the path, $h$ is the type dangerous objects. $A_{i j}$ is the dangerous objects accident rate on the path, $F_{i j}$ is dangerous objects transport vehicle accident rate on the path, $V_{\mathrm{ijh}}$ is $h$ type of dangerous objects transport round trips on the path. $r_{\mathrm{ijh}}$ is $h$ types of dangerous objects transport round trips on the path, $T_{\mathrm{ij}}$ is the number of vehicles traveling on the affected by the accidenton the path.

Companies of dangerous objects transportation follow relevant laws and regulations, but also have to maximize economic benefits. Accordingly, the road weighted valuesis introduced:

$$
W_{i j h}=1 / R_{\text {ijh }}
$$

Where $W_{\text {ijh }}$ is the road weighted values on the path.

Every path empowers the value, create a Weigh-Tables in the transition probability of ant colony algorithm, the antsincrease the value of inspired factors to choose path. The improved transition probability model is

$$
P_{i j}^{k}(t)=\left\{\begin{array}{cl}
\frac{\left[\tau_{i j}(t)\right]^{\alpha}\left[\eta_{i j}(t)\right]^{\beta}\left[w_{i j h}(t)\right]^{\gamma}}{\sum_{s \in \text { allowed }}\left[\tau_{i s}(t)\right]^{\alpha}\left[\eta_{i s}(t)\right]^{\beta}\left[w_{i s h}(t)\right]^{\gamma}} & j \in \text { allowed }_{k}, \text { (3) } \\
0 & \text { otherwise, }
\end{array}\right.
$$

Where $P_{\mathrm{ij}}$ is transition probability of ant $k(k=1,2, \ldots \ldots \mathrm{m}) \cdot \eta_{\mathrm{ij}}$ reflectsthe extent inspired of the shift. $\tau_{\mathrm{ij}}$ is pheromone trace strength of path. $\alpha(\alpha \geq 0)$ is the relative importance of trajectory. $\beta(\beta \geq 0)$ is the relative importance of visibility. $\gamma(\gamma \geq 1)$ is the appropriate value determined through experiment. allowed $_{\mathrm{k}}$ is the possible locationswhich $\mathrm{k}$-ant can select.t is time of vehicle on the path.

When transportation of dangerous objects is prohibited from $i$ to $j, R_{\mathrm{ijh}}$ assignshigh value after testing, and dangerous objects transport vehicles cannot go through the path.

\section{Program of route optimization}

Ant colony algorithm programmingby MATLABinclude: distance matrix generation, random selection starting point, generationright of way, transition probability calculation, constraints, recordingthe iteration the longest and the shortest route, pheromone update, finding the shortest route.

\subsection{Distance matrix generation}

Customer nodes number is from 1 to n,dangerous objects logistics center number is $0,\left(x_{\mathrm{i}}, y_{\mathrm{i}}\right)$ is the coordinate of customer nodes or dangerous objects logistics center.d( $i, j)$ is the distance,whichcan be expressed by the following equations:

$$
d(i, j)=\sqrt{\left(x_{i}-x_{j}\right)^{2}+\left(y_{i}-y_{j}\right)^{2}}
$$

Where $(i, j)$ is equal tod(j, $i)$. If $i$ is equal to $j, d(i, j)$ is equal to $\infty . d(i, j)$ is the column $i$ and the rowjelements of matrix $D_{\mathrm{ij}}$.

\subsection{Random selection startpoint}

We set random matrix $\mathrm{r}$, which isint32(unifrnd $\left(1, \mathrm{n}, N C_{\max }, \mathrm{m}\right)$ ). $\mathrm{n}$ is the number of customer nodes, $N C_{\max }$ is the maximum number of cycles, $m$ represents the number of ants. int32(unifrnd $(1,8,50,8))$ is randomly generated50×8dimensional integer matrix. Each line represents a starting 
point of a cycle of mants. Each iteration $m$ ants randomly selected m start points, Random selection can be more efficient test algorithm, andeffectively find the optimal solution.

Randpos =int32(unifrnd $(1,8,50,8)$ )

$\operatorname{Tabu}(:, 1)=(\operatorname{Randpos}(1,1: \mathrm{m}))$

\subsection{Right of way}

Considering transportation potentialdanger,we introduce right of wayto minimize the harm if an accident occurs.

\%right of waycalculation

fori=1:n

for $j=1: n$

ifi $\sim=\mathrm{j}$

$H(\mathrm{i}, \mathrm{j})=\sum_{i=1}^{n} \sum_{j=1}^{n} R_{\mathrm{ijh}} ; \% H$ is right of way

\section{Test results}

There is a set of 7 customer nodesof general cargo transport [Table1], collected by Li Ning[11] which areused in the literature.

Table 1Coordinate and cargo of general cargo transport.

\begin{tabular}{ccc}
\hline code number & coordinate & cargo $\left(\boldsymbol{g}_{\mathbf{i}}\right)$ \\
\hline 0 & $(18,54)$ & 0 \\
1 & $(22,60)$ & 0.89 \\
2 & $(58,69)$ & 0.14 \\
3 & $(71,71)$ & 0.28 \\
4 & $(83,46)$ & 0.33 \\
5 & $(91,38)$ & 0.21 \\
6 & $(24,42)$ & 0.41 \\
7 & $(18,40)$ & 0.57 \\
\hline
\end{tabular}

Description of problems: 0 is central warehouse.Vehicle capacity $q$ is 1.0 .Three vehicles assume all transportation tasks.

Parameters used: In all runs the parameters were set as: $\alpha$ is equal1, $\beta$ is equal5, $\rho$ is equal 0.1 , Computational result of general cargo transport vehicle routing is shown in Fig.1.

The above parameters of general cargo transport vehicle routing problem is unchanged, Segment weighting adjustment factor $\gamma$ is 4 , and introduce dangerous objectsrisk-degree of road[Table 2].

Table 2Dangerous objects risk-degree of $\operatorname{road}\left(10^{-4}\right)$.

\begin{tabular}{|c|c|c|c|c|c|c|c|c|}
\hline $\begin{array}{c}\text { task } \\
\text { poìntsi } \\
\text { risk } \\
\text { task } \\
\text { degree } \\
\text { points j }\end{array}$ & 0 & 1 & 2 & 3 & 4 & 5 & 6 & 7 \\
\hline 0 & $\mathbf{1 0}^{7}$ & 12564.3 & 1018.5 & $\mathbf{1 0}^{7}$ & 624.4 & 11891.1 & 1601.1 & 624.4 \\
\hline 1 & 12564.3 & $\mathbf{1 0}^{\mathbf{7}}$ & 1397.5 & 4388.9 & 16845 & $\mathbf{1 0}^{\mathbf{7}}$ & 1795.4 & 2489.5 \\
\hline 2 & 1018.5 & 1397.5 & $\mathbf{1 0}^{\mathbf{7}}$ & 1132.6 & 4094 & $\mathbf{1 0}^{\mathbf{7}}$ & 6058.8 & 8565.6 \\
\hline 3 & $\mathbf{1 0}$ & 4388.9 & 1132.6 & $\mathbf{1 0}^{\mathbf{7}}$ & $\mathbf{1 0}^{\mathbf{7}}$ & 1209.5 & 5303 & $\mathbf{1 0}^{\mathbf{7}}$ \\
\hline 4 & 624.4 & 16845 & 4094 & $\mathbf{1 0}^{\mathbf{7}}$ & $\mathbf{1 0}^{\mathbf{7}}$ & 9417.7 & 3686.8 & $\mathbf{1 0}^{\mathbf{7}}$ \\
\hline 5 & 11891.1 & $\mathbf{1 0}^{\mathbf{7}}$ & $\mathbf{1 0}^{\mathbf{7}}$ & $\mathbf{1 2}^{7} 209.5$ & 9417. & $\mathbf{1 0}^{\mathbf{7}}$ & 3906 & $\mathbf{1 0}^{\mathbf{7}}$ \\
\hline
\end{tabular}




\begin{tabular}{|c|c|c|c|c|c|c|c|c|}
\hline & & & & & 7 & & & \\
\hline 6 & 1601.1 & 1795.4 & 6058.8 & 5303 & 3686. & 3906 & $\mathbf{1 0}^{\mathbf{7}}$ & 1658 \\
& & & & & 8 & & & \\
\hline 7 & 624.4 & 2489.5 & 8565.6 & $\mathbf{1 0}^{7}$ & $\mathbf{1 0}^{\mathbf{7}}$ & $\mathbf{1 0}^{\mathbf{7}}$ & 1658 & $\mathbf{1 0}^{\mathbf{7}}$ \\
\hline
\end{tabular}

If $i$ is equal to $j$, overall risk-degree of dangerous objects is $10^{7}$ on path $(i, j)$, which means ants does not go through the path $(i, j)$. If others overall risk-degree of path is $10^{7}$, for example, from 2 to 5 on the path, dangerous objects transportation vehicle is prohibited according to regulations. Other data in table 2 are collected by Xiangao Wang [12].

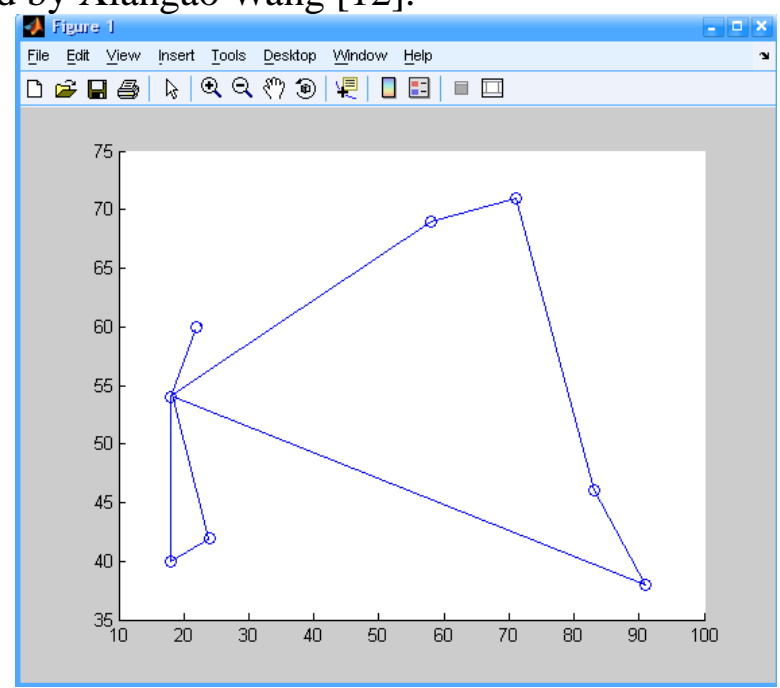

Fig. 1.General cargo transport vehicle routing simulation map.

Computational results of dangerous objects transportation vehiclerouting is shown in Fig. 2. Comparing figure 1 and figure 2, withthe same parameters, considering overallrisk-degree of dangerous objects, the optimalroute ofdangerous objects transportation vehicle is different from the optimal route of

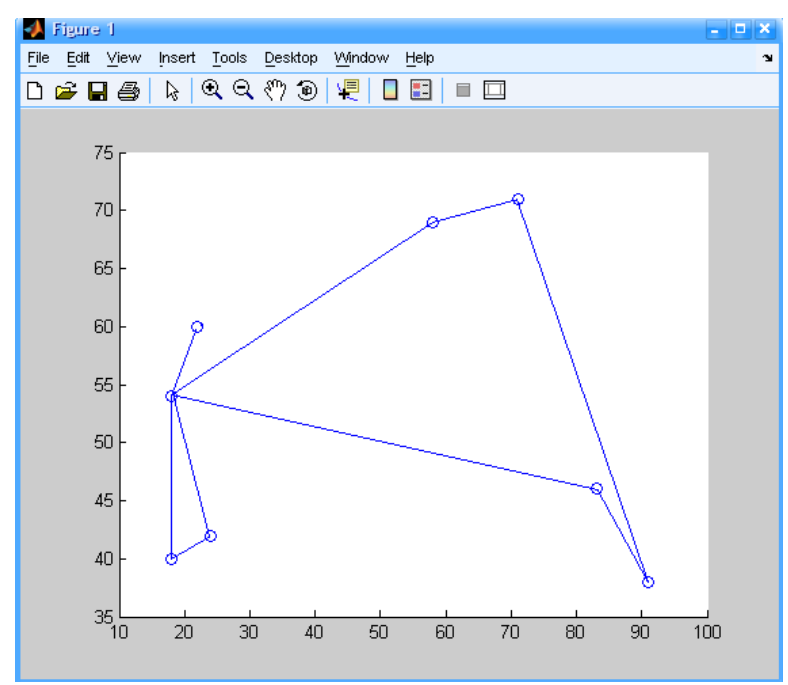

Fig. 2.Dangerous objects transportvehiclerouting simulation map.

general cargo transport vehicle. The length of dangerous objects transportation vehicle route is 219.4279, which is longer than the length of general cargo transport routes217.8135. As shownin our case study,improved ant colony algorithm successfully apply in dangerous objects transport vehicle route.

\section{Conclusions}

In this study, we have introduced road weighted valuesin the transition probability of ant colony algorithm, before select path, the ants consider the impact of the degree of road risk. 
Ant colony algorithm of dangerous objectstransport route-optimization programs with MATLAB,The route of application ant colony algorithm program of dangerous objectstransport is longer than generous goodstransport, which is consistent with the actual. The integrated modeling of these considerations is atopic worthy of further research.

\section{Acknowledgements}

Thanks are due to anonymous referees for their valuablecomments.

\section{References}

[1] John E. Bella, Patrick R. McMullenb. Ant colony optimization techniques for the vehicle routing problem.Advanced Engineering Informatics,18,pp.41-48, 2004.

[2] ArdavanAsef-Vaziri, MortezaKazemi, KouroshEshghi, Maher Lahmar.An ant colony system for enhanced loop-based aisle-network design.European Journal of Operational Research,207,pp.110-120,2010.

[3] S.R. Balseiro, I.Loiseau, J.Ramonet.An Ant Colony algorithm hybridized with insertion heuristics for the Time Dependent Vehicle Routing Problem with Time Windows.Computers \&OperationsResearch ,38,pp.954-966,2011.

[4]Christopher L. Fleming, tanley E. Griffis, John E. Bell. The effects of triangle inequality on the vehicle routing problem. European Journal of Operational Research, 224,pp.1-7,2013.

[5]Abkowitz, M; Cheng, P D. Developing a risk/cost framework for routing truck movements of hazardous materials. Accident analysis and prevention, 20,pp.39-51,1988.

[6]P. Leonelli, S. Bonvicini, G. Spadoni. Hazardous materials transportation: a risk-analysis-based routing methodology. Journal of Hazardous Materials, 71,pp.283-300,2000.

[7]WL Ng, SCH Leung, JKP Lam and SW Pan. Petrol delivery tanker assignment and routing: a case study in Hong Kong. Journal of the Operational Research Society ,59,pp.1191-1200,2008.

[8] Li Zhi, GuoHaixiang, LiuLonghui.Resolving Single Depot Vehicle Routing Problem with Artificial Fish Swarm Algorithm. 3rd International Conference on Swarm Intelligence (ICSI),2012, Shenzhen.

[9] LiRongrong, LeungYee, HuangBoet al.A genetic algorithm for multiobjective dangerous goods route planning. INTERNATIONAL JOURNALOF GEOGRAPHICAL INFORMATION SCIENCE,27(6),pp.1073-1089,2013.

[10]Rijling, J. K., Van Hengel, W., \&Houben, R. J.A framework forrisk evaluation. Journal of Hazardous Materials,43,pp.245-261,1995.

[11] LiNing, Zou Tong, Sun De-bao. Particle Swarm Optimization forVehicle Routing Problem with Time Windows.Systems Engineering -Theory \& Practice,4,pp.130-135,2004.

[12]XiangaoWang.Transportationof DangerousGoodsRisk Assessment.Master's thesis. China: Chang,an University;2006. 